\title{
Internal carotid artery dissection following blunt head trauma: a pediatric case report and review of the literature
}

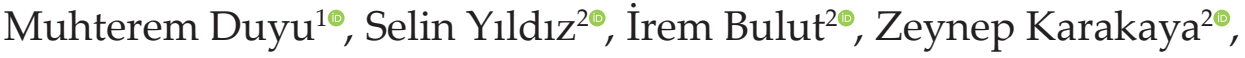 \\ Ayşenur Buz ${ }^{3 \oplus}$, Gülçin Bozbeyoğlu ${ }^{3 \oplus}$ \\ ${ }^{1}$ Division of Pediatric Intensive Care Unit, ${ }^{2}$ Department of Pediatrics and ${ }^{3}$ Department of Radiology, Istanbul Medeniyet University, \\ Göztepe Training and Education Hospital, İstanbul, Turkey.
}

\begin{abstract}
Background. Internal carotid artery dissection (ICAD) is a rare but potentially devastating complication after trauma in the pediatric age group. The diagnosis of traumatic dissection is difficult and is usually recognized only when ischemic symptoms appear. We report a pediatric patient with ICAD due to blunt cerebrovascular injury (BCVI).

Case. A 14-year-old boy suffered major trauma due to a motor vehicle accident. When the first aid team reached the accident site, he was intubated because of his low Glasgow Coma Score (GCS) and then transported to the nearest emergency department. Cranial computed tomography (CT) showed multiple fractures at the skull base and independent bone fragments in both carotid canals. On the 6th day; a brain magnetic resonance imaging (MRI) was performed to detect diffuse axonal injury. There was a loss of signal in the left internal carotid artery (LICA) tract but the limitation of diffusion was not associated with the same side, conversely there was a limitation of diffusion on the other side, affecting a very large area. CT angiography was performed in order to detect a filling defect and showed dissection in the LICA. The patient did not have any specific neurological symptoms associated with ICAD. Low-dose aspirin was utilized as anticoagulant therapy. On the 25th day of admission, the patient's GCS was 14, neurologic examination showed no difference between the right and left sides. He was discharged on the 55th day of the accident and was walking without support.
\end{abstract}

Conclusion. Our patient was a rare case in pediatrics due to having a clinically silent form of ICAD. It is very difficult to diagnose ICAD dissection during the early phase in cases with BCVI accompanied by multiple trauma. Even in the absence of typical neurological deficit, the possible presence of ICAD should be explored in patients with cranial fractures encompassing the skull base.

Key words: blunt cerebrovascular injury, carotid artery dissection, pediatric intensive care.

Blunt cerebrovascular injury $(\mathrm{BCVI})$ is a rare but potentially devastating complication after trauma, and accounts for approximately 1.5$3.5 \%$ of patients admitted to trauma centres. ${ }^{1}$ Internal Carotid artery dissections (ICAD) are rare, most of them occur spontaneously but around $4 \%$ are secondary to trauma. ${ }^{2}$ These statistics are for adult trauma patients and such traumas are rare in the pediatric age group.

$\triangle \quad$ Muhterem Duyu

drmuhteremduyu@gmail.com

Received 19th April 2020, revised 12th June 2020, accepted 5th August 2020.
Dissection can be a diagnostic challenge due to the complexity of the clinical examination in children and delayed onset of symptoms in many cases. Usually, patients cannot be diagnosed until ischemic symptoms appear. ${ }^{3}$

Despite its low incidence, dissecting injury of the supraaortic vessels (carotid artery, vertebral artery etc.) is an important cause of ischemic stroke in children, associated with significant morbidity and mortality rates up to $20 \%{ }^{4}$ Arterial dissection may be caused by direct trauma to the artery, hyperextension-rotation of the head with stretching of vessels, basal skull or mandibular fractures, chest-head injuries 
with carotid stretching, and blunt intra-oral trauma. ${ }^{5-8}$

In this case report, we report a rarely seen pediatric case of ICAD development due to blunt head trauma. Although the ICAD developed on the left side, ischemic areas were predominantly located on the right cerebral hemisphere due to diffuse axonal injury (DAI) which was secondary to traumatic brain injury (TBI).

\section{Case Report}

A previously healthy 14-year-old male patient suffered major trauma due to a motor vehicle accident. The mechanism of this major trauma was a tractor rollover during harvest. When the first aid team reached the accident site, the boy was lying still on the ground and was immediately intubated because of his low Glasgow Coma Score (GCS) (E1M4V1). $\mathrm{He}$ was immobilized and then transported to the nearest emergency site. At his arrival, GCS was 6 (E1M4V1) and all immediately necessary interventions were performed by the emergency team. His cranial, cervical, thoracic and abdominal imaging studies were performed after he was stabilized, and then he was transported to our pediatric intensive care unit (PICU).

At his examination in the PICU, the patient was intubated, bilateral pupils were isochoric with positive light reflex, and all four extremities were equally moving with painful stimuli. Babinski sign was negative. Due to the patient being unconscious and intubated, a cranial nerve exam could not be performed conclusively. He was hemodynamically stable. The hemogram, arterial blood gas and biochemical parameters of the patient were normal. His thorax, abdominal and cervical computed tomography (CT) screenings were normal. In the bone window setting, cranial CT images showed multiple fractures at the base of the skull, and a bilateral styloid process fracture and hemotympanum (Fig. 1).
We noticed clear secretions in the mouth, which seemed similar to cerebrospinal fluid (CSF). These were tested with the beta-2 transferrin test, confirming our suspicions. To detect the basilar skull fracture that was causing CSF leak, a maxillofacial sinus CT was also performed. There were independent bony fragments in both carotid canals, hemorrhagic collections in the sphenoid and ethmoid sinuses, and fractured fragments of the left temporal bone causing independent bone fragments (Fig. 2).

After discontinuing all sedative medications, GCS was reevaluated. Because GCS was under 8 (E1M4V1), intracranial pressure catheterization (ICP) was performed at the bedside. He was followed-up with ICP catheter for five days to minimize TBI. Our goals were to keep ICP $<20$ $\mathrm{mmHg}$ and cerebral perfusion pressure $>55$ $\mathrm{mmHg}$. On the $6^{\text {th }}$ day of PICU follow-up, his ICP catheter was removed and his neurologic examination was checked after discontinuing sedation. His GCS was 6 (E1M4V1), pupils were isochoric, light reflex was bilateral positive, and all four extremities were moving equally.

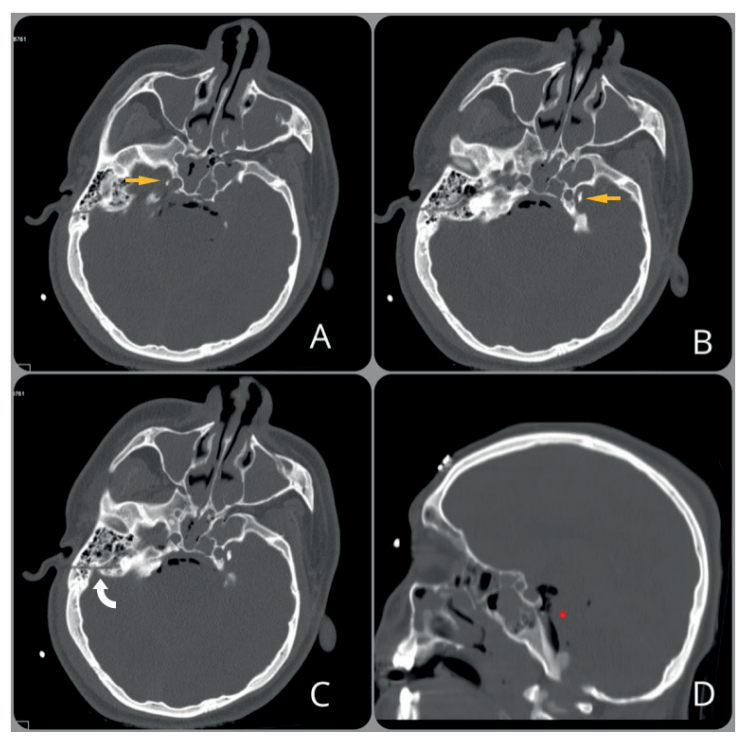

Fig. 1. Initial head CT reveals multiple fractures in skull base including lateral walls of carotid canals on both sides. On image A and B yellow arrows depict the separated osseous fragments. On image C, curved arrow shows longitudinal fracture of right temporal bone. On image D, pneumocephalus (red star) is demonstrated. 

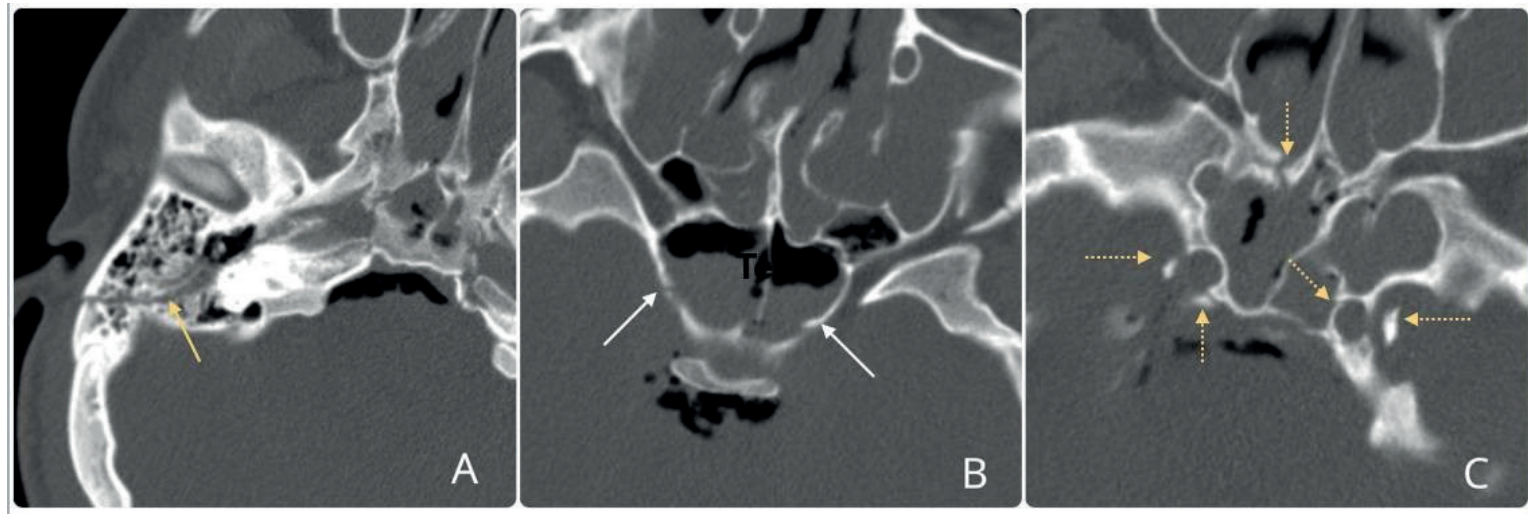

Fig. 2. On image A, fracture of the right temporal bone (yellow arrow). On image B, intense hemorrhagic collections of fractures in the sphenoid sinus and ethmoid cellulites posterior to the nasal septum and filling the secondary sinuses (white arrow), and on image C, free osseous fragment in both carotid channels (yellow arrow).

On the $6^{\text {th }}$ day after the accident, a brain magnetic resonance imaging (MRI) was performed to detect DAI. When evaluating arteries, we determined a loss of signal in the left internal carotid artery (LICA) tract but diffusion limitation area was not correlated with the region supplied by the LICA. Surprisingly, the diffusion restriction of the right hemisphere was worse than that of the left side (Fig. 3). To verify this loss of signal in the LICA tract, CT angiography was performed and revealed filling defect and presence of intimal flap on the left side of the LICA's cavernous segment and LICA's petrous part, which was verifying results with MRI screening (Fig. 4).

A detailed neurological exam could not be performed due to low GCS and intubation; nevertheless, as far as examination could show, there was no specific neurological deficit (hemiplegia, hemiparesis, facial palsy etc.) that was conclusive for left ICAD. As such, ICAD was only detected during a routine MRI performed on the 6th day of admission. Because it was late for the endovascular intervention and the use of heparin were not suitable due to trauma presence, we only used low-dose aspirin (100 $\mathrm{mg} /$ day) as an anticoagulant.

Due to his low GCS (E2M4V1), tracheostomy and percutaneous enteral gastrostomy operations were performed on the 15th day of hospital stay. His neurological state was progressively getting better. On the $25^{\text {th }}$ day of PICU stay, GCS was 14 , muscle strength was $2 / 5$ for upper extremities and $3 / 5$ for lower extremities. Surprisingly,
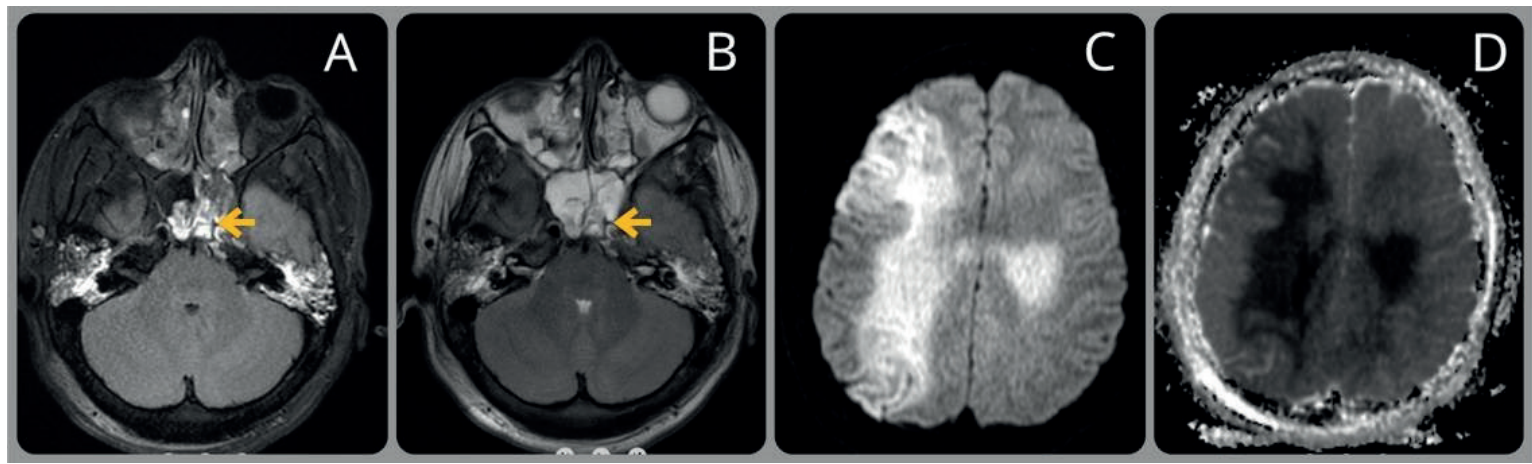

Fig. 3. Diffusion restriction in bilateral watershed areas of brain predominantly on right side is seen on image $\mathrm{C}$ and D. Retrospectively we also noticed loss of signal void on left internal carotid artery (yellow arrow) which is in favor of arterial dissection on image A and B. 


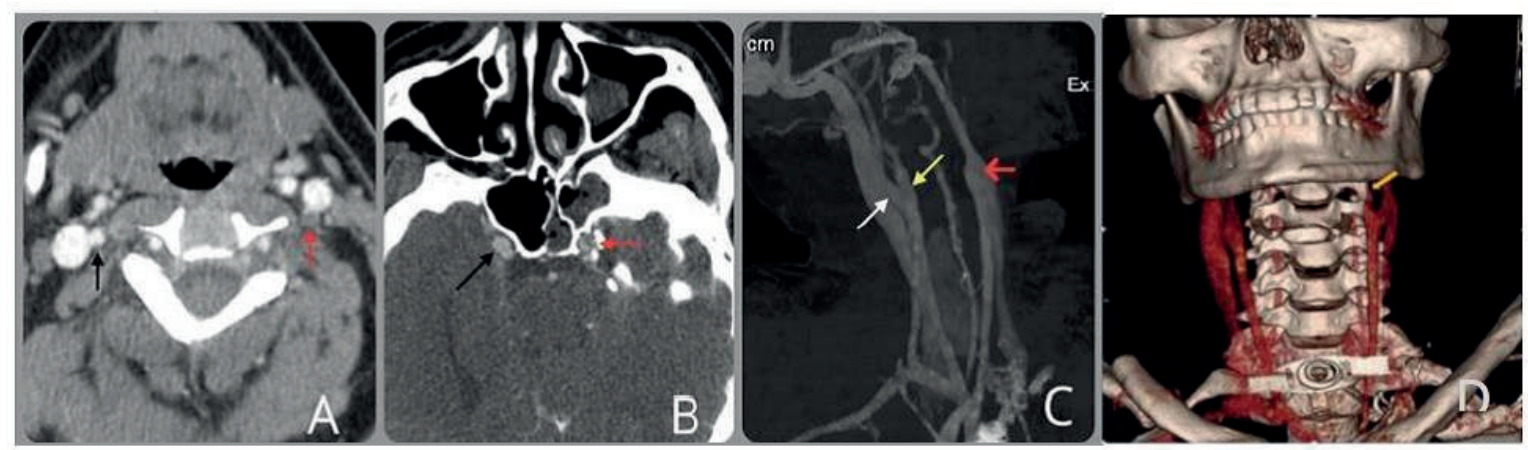

Fig. 4. CT angiography reveals filling defect in cervical portion of the left internal carotid artery (LICA), indicated with red arrow (Image A). At the level of the carotid canal, an intimal flap on the left side is demonstrated (Image B, red arrow). On maximum intensity projection images (Image C and D), it is clearly appreciated that there is no luminal enhancement at neither left common carotid artery from the level of bifurcation nor LICA (red arrows). Only external carotid artery is filled with contrast media which are highly suggestive findings for dissection of LICA. 3D CT angiography reveals filling defect in cervical portion of the LICA, indicated with yellow arrow (Image D).

there was no difference between the right and left sided neurological examinations. There were no findings of cranial nerve paralysis. Daily physiotherapy was performed. During his follow-up, muscle strength reached $4 / 5$ and $5 / 5$ for upper and lower extremities, respectively. When his GCS rose to E4M6V5 on the $51^{\text {th }}$ day of hospitalization, tracheostomy and gastrostomy were closed. As his neurological improvement was very fast he was discharged from the hospital safely on the $55^{\text {th }}$ day of the accident, and he was walking without support.

Informed consent was obtained from the patient's parents for publication.

\section{Discussion}

Traumatic carotid artery injuries (CAI) are rare; however, because of the anatomy, there may be important life-threatening complications. It occurs especially in patients with multiple trauma and direct blunt trauma to the neck. Delayed clinical presentation of traumatic ICAD may occur after weeks, months, even years following injury and it has important clinical outcomes. ${ }^{3}$ Our case demonstrates that ICAD can be easily missed during the initial trauma survey in patients with multiple trauma, especially in cases of additional TBI. Knowledge of the mechanism and risk factors of the traumatic ICAD is important because it could have consequences for the treatment of these patients. A literature review revealed 21 published cases of ICAD caused by trauma. ${ }^{6-21}$ These cases are summarized in Table I.

\section{Pathophysiology}

Dissections start with a tear in the intima/media layer of the vessel wall, after which a hematoma develops. Ischemic strokes after ICAD may occur because of thromboembolism, or, less frequently, via artery occlusion due to the hematoma in the vessel wall. ${ }^{1,22}$ Dissection can also progress in the subintimal or subadventitial layer, leading to local compression resulting in a loss of function of the adjacent cranial nerves, presenting as Horner syndrome or facial nerve palsy. ${ }^{1,22}$

Dissections may be traumatic or spontaneous. In addition to severe traumas like motor vehicle accidents, direct neck trauma and mild trauma like minor shoulder trauma can also be associated. ${ }^{12,15,20}$ Traumatic ICAD may develop in association with the following mechanisms: I- hyperextension and contralateral rotation of the head and neck, II- blunt intraoral trauma that affects the internal carotid artery at the angle of the jaw, III- laceration of the artery due to the skull base and mandible fractures, 


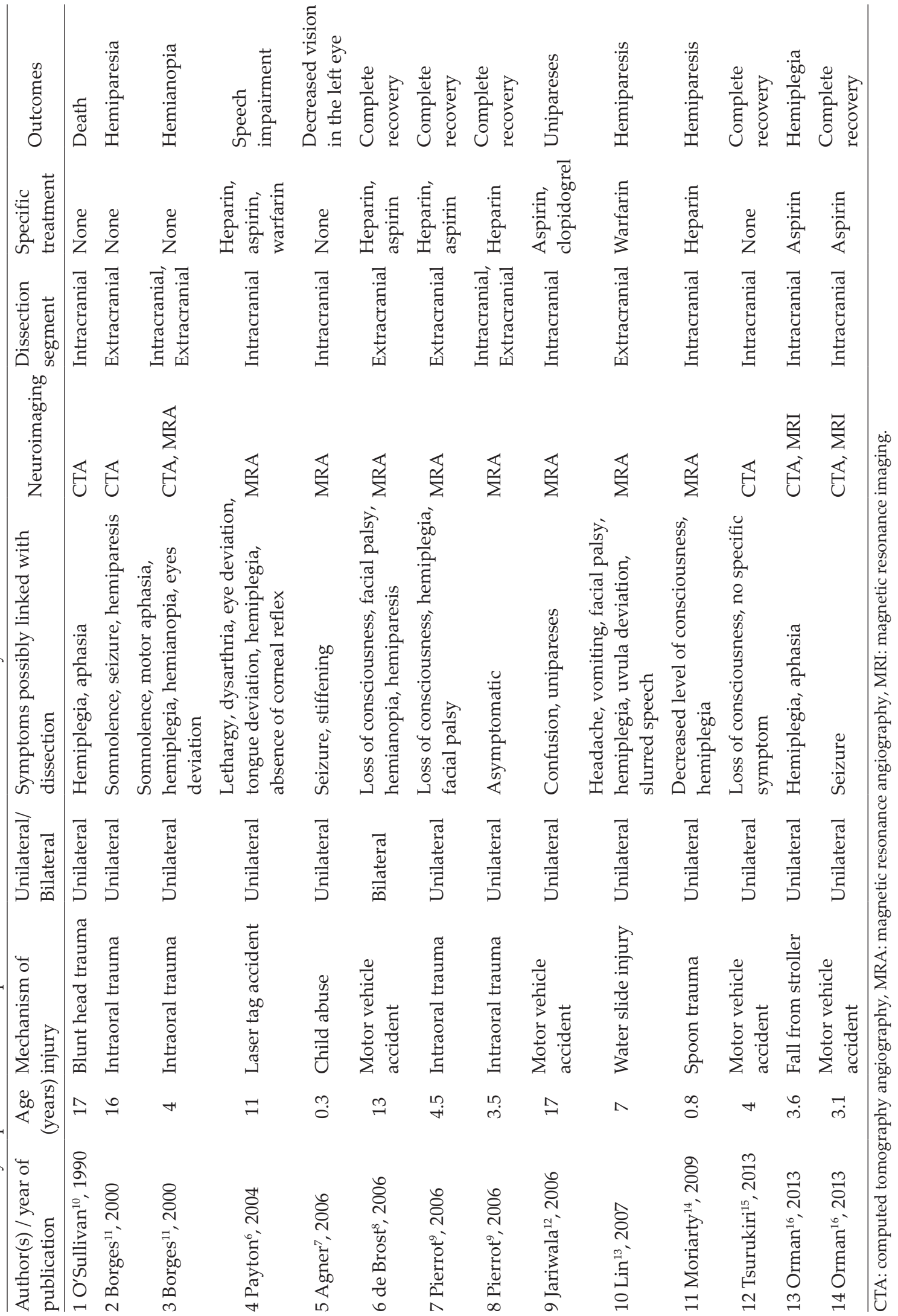




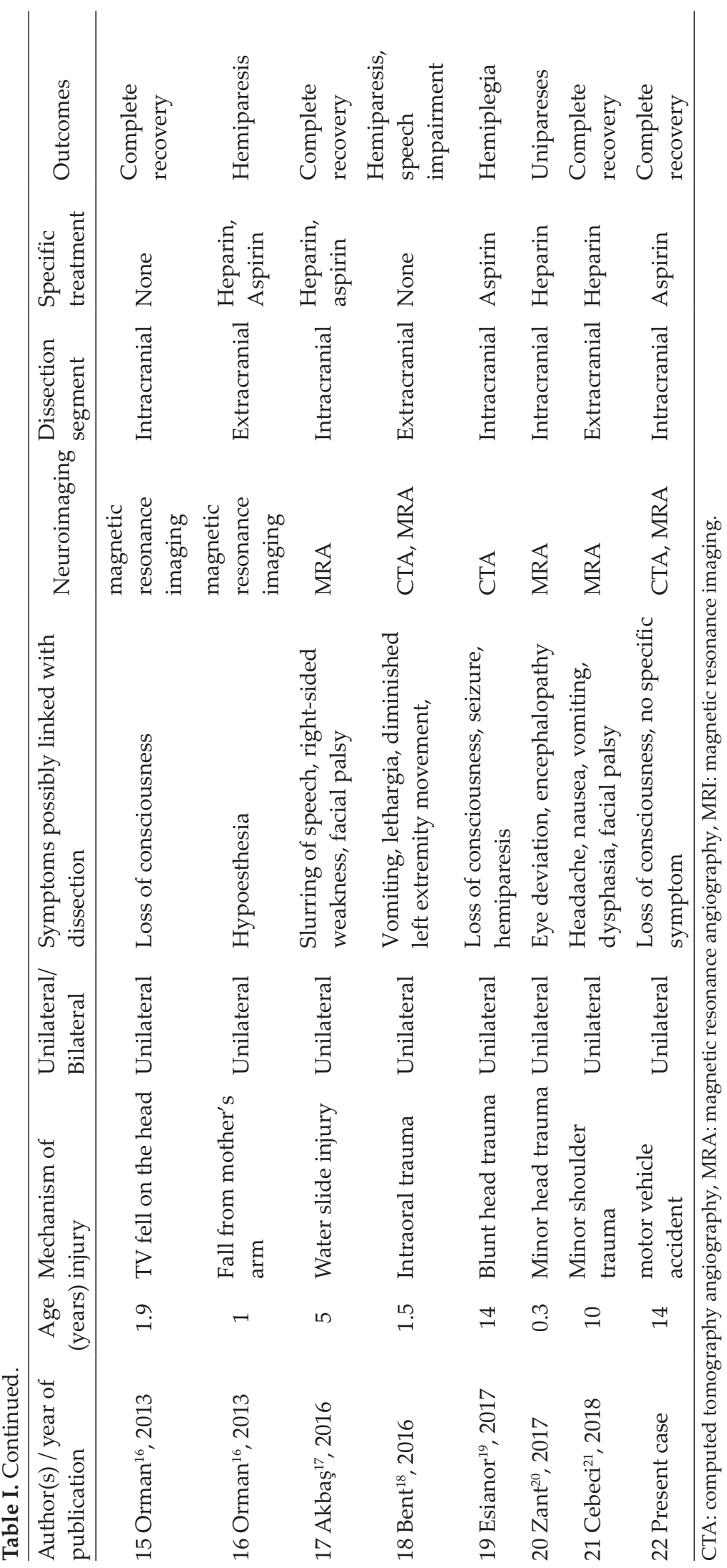


IV- a combination of head-thorax trauma with overstretching of the internal carotid artery, $\mathrm{V}$ - direct application of force to the neck. ${ }^{1}$ The frequency of ICAD is especially high in cases with accompanying carotid or petrous bone fractures in association with skull base fractures due to blunt trauma. ${ }^{23}$ We also identified a skull base fracture extending to the lateral wall of the carotid canal in our patient.

Spontaneous ICADs are more common in the intracranial region, and traumatic dissections of the ICA in the head and neck region are most frequently located in the extracranial segments of the vessel. Concerning the ICA, most of the traumatic dissections occur below the skull base. ${ }^{24-26}$ In our case, blunt trauma-related ICAD was observed in the intracranial segment of the ICA.

\section{Clinical Symptoms}

The most common signs and symptoms are hemipareses, headache, aphasia, dysphasia, cranial nerve palsy, anopsia and an altered level of consciousness. CAI may present with an ischemic event such as transient ischemic attack (TIA) or stroke before reaching the hospital. Other manifestations may include ipsilateral headache, Horner syndrome, neck pain, bruit, and tinnitus. ${ }^{27}$ In cases with traumatic mechanism, bleeding through the oral cavity, nostrils or ears can be detected as well. The diagnosis is relatively late due to the presence of other lesions related to trauma and this causes important sequalae. ${ }^{28}$ In our case, no specific neurological findings could be detected, and a detailed neurological evaluation could not be performed because of TBI and unconsciousness. In the 21-case literature review we have reported, it is evident that cranial nerve paralysis and neurological findings such as hemiplegia, hemiparesis are at the forefront. However, due to the lack of clinical findings suggestive of ICAD, the diagnosis was delayed in our case.

There is usually a significant time interval between trauma and neurological symptoms in such patients, even though some cases have been shown to have immediate findings. The interval between blunt trauma and neurological deficits can be hours, days, or even months..$^{22,29}$ Especially in children, the dissection may remain in the subadventitial layer rather than the subintimal layer, which may account for a delay in presentation. ${ }^{29}$ This delay between the moment of dissection and the occurrence of neurological symptoms is the critical factor that makes diagnosis difficult. Further, it is not at all clear that early detection would have found a treatable lesion after a stroke that is clinically evident hours or days after the injury. Reports of strokes developing after initial negative screening have been made. ${ }^{30}$ On the other hand, the time interval between injury and onset of symptoms offers the possibility of screening for $\mathrm{CAD}$ and initiating therapy before the neurological symptoms become clear.

\section{Diagnosis}

Whilst the demonstration of an initial flap, double lumen sign or string sign (areas of adjacent stenosis and dilatation) on conventional catheter angiography remains the gold standard for diagnosis of CAD; non-invasive diagnosis with duplex ultrasound (USG), CT angiography (CTA) or MR angiography (MRA) is being increasingly used. Duplex USG has poor vision of the intracranial aspects of a dissection and it also provides limited information about small intimal tears and it is difficult to interpret when there is a hematoma in the neck. ${ }^{1}$

CTA is prominently used as diagnostic modalities for the detection of BCVI, especially considering the acute situation in the emergency room of severely injured patients. Further development of CT scanners (>64 slices) shows comparable results in sensitivity to MRA scans. ${ }^{2}$ However, to get additional information about a possible CVI, it can be necessary to include CTA into the protocol of the whole-body CT. To rule out patients that need an additional CTA, the modified "Denver Screening Criteria" (Table II) can be helpful. ${ }^{32}$ 
Table II. Modified Denver screening criteria for BCVI.

\begin{tabular}{ll}
\hline Sign of BCVI & Risk factors for BCVI \\
\hline Arterial bleeding & High-energy trauma mechanism combined with: \\
Cervical hematoma & Le Fort II/III fractures \\
Focal neurological deficiency & Cervical spine fractures \\
Neurological findings not matching with CT findings & Basilar skull fracture with carotid canal involvement \\
Ischemic insult seen on a secondary CT scan & Diffuse axonal injury with GCS $\leq 6$ \\
Cervical heart murmur & Near hanging with anoxic brain injury \\
& Combination of TBI and thorax trauma \\
\hline
\end{tabular}

BCVI: blunt cerebrovascular injury, CT: computed tomography, GCS: Glasgow coma scale, TBI: traumatic brain injury.

Imaging with an MRI scan combined with the MRA is considered to be the gold standard for the detection of carotid artery dissections. ${ }^{32}$ It determines dissections in up to $99 \%$ of cases and provides additional information about concomitant injuries (i.e. diffuse axonal injuries, acute infarction, fractures, etc.). ${ }^{2}$ But it also has certain limits, especially during the diagnosis of critically injured patients with trauma. Besides the long duration of the scan, on the one hand, there exist restrictions in the use of potentially life supporting devices (i.e. pacemakers/ironbased metal implants, etc.), while on the other hand, there is a possibility that MRI availability is limited, especially in smaller trauma centers. ${ }^{31}$ The choice of the screening method may change with regard to the clinical setting and patient condition. In our 16-case literature review, it was determined that diagnosis was most commonly performed via MRA. In our case however, the diagnosis was confirmed via CTA after suspicious signal-loss findings were identified in MRI imaging.

\section{Therapeutic options}

In general, asymptomatic patients with lowgrade dissections are treated conservatively with medical management and close imaging. There are several therapeutic options to treat cerebrovascular dissections, including open surgery, endovascular management (stenting, endovascular thrombolysis, thrombectomy), thrombolysis, anticoagulant and antithrombotic therapy. ${ }^{31}$
Surgical options are difficult and risky in the acute phase of the dissection. ${ }^{33}$ This is why they usually do not belong in the first line of therapy in the acute phase. However, there are certain cases where operative treatment is necessary (i.e. to restore the blood flow in a case of distinct tear of the vessels or complete occlusions without ischemic intracranial lesions). ${ }^{31}$

The literature on the endovascular treatment (stenting) of traumatic ICAD is still limited to small series based on adult patients with relatively short follow-up. A proportion of the dissections occur at the skull base or in the intracranial region, and are therefore inaccessible to surgical or endovascular therapy. However, recent studies of stenting for CAD show excellent early and one-year patency rates and a low major adverse event rate. ${ }^{34}$ Endovascular local thrombolysis is thought to be useful especially to prevent thromboembolic cerebral insult, but they still present high risks in the setting of a severely injured patient with multiple fractures or TBI, as presented in our case. $^{2,31}$

Antithrombotic or antiplatelet treatments are recommended in patients with neurological symptoms; whereas endovascular intervention is suggested only when the neurological status of a patient deteriorates under conservative medical treatment. ${ }^{35}$ Antithrombotic treatment consists of either anticoagulation, typically with intravenous heparin followed by warfarin, or antiplatelet therapy with aspirin. Anticoagulation is typically preferred over 
antiplatelet therapy in severe stenosis or pseudoaneurysm -assuming that it prevents thromboembolic complications more effectively. Patients who are unable to receive anticoagulants were treated with antiplatelet therapy. ${ }^{36}$ In adults, anticoagulation therapy is suggested for 3 to 6 months to prevent injuries progressing to a higher injury grade, and to reduce the number of strokes. ${ }^{36}$ Thrombolysis regimens, including recombinant tissue plasminogen activator or urokinase, are used to restore blood flow in some cases. But these approaches require an early diagnosis. The rationale of antithrombotic therapy is that the mechanism of the majority of strokes in CAD is due to thromboembolism. ${ }^{36}$

In the pediatric population, the treatment of CAD is still controversial and more studies are needed. The use of antiplatelet therapy is even more controversial for patients who suffer a stroke following an intracranial dissection because of a very real risk of intracranial haemorrhage. ${ }^{21}$ Unfortunately, patients who suffered from blunt carotid injuries typically have closed head injuries, solid organ injuries, and/or pelvic fractures that prevent the use of early anticoagulation. When other concomitant injuries are present, then the optimal type and time of treatment should be considered with an interdisciplinary approach. ${ }^{37}$ Pandey at al. ${ }^{38}$ recommended 3 months of anticoagulation therapy and then antiplatelet therapy. In our case, as there were no specific symptoms and the diagnosis was made in the later period, we only used low-dose aspirin as an antiaggregant therapy. Anticoagulant therapy was not suitable for our patient due to multiple trauma.

\section{Prognosis}

Blunt trauma-related ICAD is known to carry a high rate of devastating neurologic morbidity $(60 \%)$ and mortality (19-43\%). Interestingly, a significant number of patients (66-73\%) may be asymptomatic upon initial presentation, developing delayed neurologic symptoms anywhere from 1 hour to 7 days after injury. 8,9,22,29
CAD patients $(33.7 \%)$ may present with an ischemic event at the time of presentation. As the severity of vessel injury worsens, stroke and mortality rates consequently increase ${ }^{31}$ In our review of the 21 pediatric patients with ICAD, $47.6 \%(n=11)$ of cases did not have neurological symptoms at admission. The death rate was $4.8 \%(\mathrm{n}=1)$ and $40 \%(\mathrm{n}=8)$ of survivors had a complete recovery. However, it must be noted that ICAD was accompanied by TBI -which may have influenced neurological outcomes.

It is difficult to diagnose ICAD in the early phase in patients with TBI accompanied by multiple trauma. Even in the absence of typical neurological deficit (as was the case in our patient), the possible presence of ICAD should be explored in those with cranial fractures encompassing the skull base. Regarding therapeutic options, bleeding risk is one of the most challenging aspects of treatment considerations, especially in the presence of multiple trauma (i.e. pelvic fractures, or haemorrhagic traumatic contusion).

\section{REFERENCES}

1. Miller PR, Fabian TC, Croce MA, et al. Prospective screening for blunt cerebrovascular injuries: analysis of diagnostic modalities and outcomes. Ann Surg 2002; 236: 386-395.

2. Jansen G, Popp J, Dietrich U, Mertzlufft F, Bach F. Traumatic dissection of the carotid artery: challenges for diagnostics and therapy illustrated by a case example. Anaesthesist (Berl) 2013; 62: 817-823.

3. Davis JW, Holbrook TL, Hoyt DB, Mackersie RC, Field TO Jr, Shackford SR. Blunt carotid artery dissection: incidence, associated injuries, screening, and treatment. J Trauma 1990; 30: 1514-1517.

4. Bowen MD, Burak CR, Barron TF. Childhood ischemic stroke in a nonurban population. J Child Neurol 2005; 20: 194-197.

5. Cheon JE, Kim IO, Kim WS, Hwang YS, Wang KC, Yeon KM. MR diagnosis of cerebellar infarction due to vertebral artery dissection in children. Pediatr Radiol 2001; 31: 163-166.

6. Payton TF, Siddiqui KM, Sole DP, McKinley DF Traumatic dissection of the internal carotid artery. Pediatr Emerg Care 2004; 20: 27-29. 
7. Agner C, Weig SG. Arterial dissection and stroke following child abuse: case report and review of the literature. Childs Nerv Syst 2005; 21: 416-420.

8. de Borst GJ, Slieker MG, Monteiro LM, Moll FL, Braun KP. Bilateral traumatic carotid artery dissection in a child. Pediatr Neurol 2006; 34: 408411.

9. Pierrot S, Bernardeschi D, Morrisseau-Durand MP, Manach Y, Couloigner V. Dissection of the internal carotid artery following trauma of the soft palate in children. Ann Otol Rhinol Laryngol 2006; 115: 323329.

10. O'Sullivan RM, Robertson WD, Nugent RA, Berry K, Turnbull IM. Supraclinoid carotid artery dissection following unusual trauma. AJNR Am J Neuroradiol 1990; 11: 1150-1152.

11. Borges G, Bonilha L, Santos SF, et al. Thrombosis of the internal carotid artery secondary to soft palate injury in children and childhood. Report of two cases. Pediatr Neurosurg 2000; 32: 150-153.

12. Jariwala SP, Crowley JG, Roychowdhury S. Trauma-induced extracranial internal carotid artery dissection leading to multiple infarcts in a young girl. Pediatr Emerg Care 2006; 22: 737-742.

13. Lin JJ, Chou ML, Lin KL, Wong MC, Wang HS. Cerebral infarct secondary to traumatic carotid artery dissection. Pediatr Emerg Care 2007; 23: 166168.

14. Moriarty JM, Lukas C, Rossler L, Thiels C, Drescher R. Carotid artery dissection following a minor household accident in a 10-month-old child. Ir J Med Sci 2009; 178: 535-539.

15. Tsurukiri J, Nomura O, Okita T, Fukami S, Ikeda Y. Internal carotid artery dissection at the supraclinoid portion after severe traumatic head injury in a child. Signa Vitae 2013; 8: 67-69.

16. Orman G, Tekes A, Poretti A, Robertson C, Huisman TAGM. Posttraumatic carotid artery dissection in children: not to be missed! J Neuroimaging 2014; 24 : 467-472.

17. Akbas Y, Arhan E, Serdaroglu A, Nazlıel B. Intracranial internal carotid artery dissection following waterslide use: the first case report. Childs Nerv Syst 2016; 32: 411-413.

18. Bent C, Shen P, Dahlin B, Coulter K. Blunt intraoral trauma resulting in internal carotid artery dissection and infarction in a child. Pediatr Emerg Care 2016; 32: 534-535.

19. Esainor BI, Haider AS, Engelhardt MI, et al. Intracranial ischemic infarct due to blunt force trauma in a high school football player. Cureus 2017; 9: e1659.
20. Zant R, Melter M, Doerfler C, et al. Carotid artery dissection with associated territory stroke after a minor head trauma in a healthy 4-month-old child. Pediatr Emerg Care 2017; 33: e177-e179.

21. Cebeci D, Arhan E, Demir E, et al. Internal carotid artery dissection without intracranial infarct following a minor shoulder trauma: the second pediatric case and review of the literature. J Clin Neurosci 2018; 56: 172-175.

22. Kraus RR, Bergstein JM, DeBord JR. Diagnosis, treatment and outcome of blunt carotid arterial injuries. Am J Surg 1999; 178: 190-193.

23. McKevitt EC, Kirkpatrick AW, Vertesi L, Granger R, Simons RK, Chir B. Identifying patients at risk for intracranial and extracranial blunt carotid injuries. Am J Surg 2002; 183: 566-570.

24. Chamoun RB, Mawad ME, Whitehead WE, Luerssen TG, Jea A. Extracranial traumatic carotid artery dissections in children: a review of current diagnosis and treatment options. J Neurosurg Pediatr 2008; 2: 101-108.

25. Mohan IV. Current optimal assessment and management of carotid and vertebral spontaneous and traumatic dissection. Angiology 2014; 65: 274283.

26. Downer J, Nadarajah M, Briggs E, Wrigley $P$, McAuliffe W. The location of origin of spontaneous extracranial internal carotid artery dissection is adjacent to the skull base. J Med Imaging Radiat Oncol 2014; 58: 408-414.

27. Richard SA, Zhang CW, Wu C, Ting W, Xiaodong $X$. Traumatic penetrating neck injury with right common carotid artery dissection and stenosis effectively managed with stenting: a case report and review of the literature. Case Rep Vasc Med 2018; 2018: 4602743.

28. Lee TS, Ducic Y, Gordin E, Stroman D. Management of carotid artery trauma. Craniomaxillofac Trauma Reconstr 2014; 7: 175-189.

29. Pozzati E, Giuliani G, Poppi M, Faenza A. Blunt traumatic carotid dissection with delayed symptoms. Stroke 1989; 20: 412-416.

30. Stein DM, Boswell S, Sliker CW, Lui FY, Scalea TM. Blunt cerebrovascular injuries: does treatment always matter? J Trauma 2009; 66: 132-144.

31. Crönlein M, Sandmann GH, Beirer M, Wunderlich S, Biberthaler P, Huber-Wagner S. Traumatic bilateral carotid artery dissection following severe blunt trauma: a case report on the difficulties in diagnosis and therapy of an often overlooked life-threatening injury. Eur J Med Res 2015; 20: 62. 
32. Vertinsky AT, Schwartz NE, Fischbein NJ, Rosenberg J, Albers GW, Zaharchuk G. Comparison of multidetector CT angiography and MR imaging of cervical artery dissection. AJNR Am J Neuroradiol 2008; 29: 1753-1760.

33. Rao AS, Makaroun MS, Marone LK, Cho JS, Rhee R, Chaer RA. Long-term outcomes of internal carotid artery dissection. J Vasc Surg 2011; 54: 370-375.

34. DuBose J, Recinos G, Teixeira PG, Inaba K, Demetriades D. Endovascular stenting for the treatment of traumatic internal carotid injuries: expanding experience. J Trauma 2008; 65: 1561-1566.
35. Galyfos G, Filis K, Sigala F, Sianou A. Traumatic carotid artery dissection: a different entity without specific guidelines. Vasc Specialist Int 2016; 32: 1-5.

36. Peng J, Liu Z, Luo C, et al. Treatment of cervical artery dissection: antithrombotics, thrombolysis, and endovascular therapy. Biomed Res Int 2017; 2017: 3072098.

37. Arthurs ZM, Starnes BW. Blunt carotid and vertebral artery injuries. Injury 2008; 39: 1232-1241.

38. Pandey AS, Hill E, Al-Holou WN, et al. Management of pediatric craniocervical arterial dissections. Childs Nerv Syst 2015; 31: 101-107. 